\title{
Application of the Feshbach-resonance management to a tightly confined Bose-Einstein condensate
}

\author{
G. Filatrella, ${ }^{1,2}$ B.A. Malomed ${ }^{3}$ and L. Salasnich ${ }^{4}$ \\ ${ }^{1}$ Department of Biological and Environmental Sciences, \\ University of Sannio, via Port'Arsa 11, 82100 Benevento, Italy \\ ${ }^{2}$ CNR-INFM, Regional Laboratory SUPERMAT, via S. Allende, 84081 Baronissi, Italy \\ ${ }^{3}$ Department of Physical Electronics, School of Electrical Engineering, \\ Faculty of Engineering, Tel Aviv University, Tel Aviv 69978, Israel \\ ${ }^{4}$ CNR-INFM, CNISM, and Department of Physics "Galileo Galilei", University of Padua, 35122 Padua, Italy
}

\begin{abstract}
We study suppression of the collapse and stabilization of matter-wave solitons by means of timeperiodic modulation of the effective nonlinearity, using the nonpolynomial Schrödinger equation (NPSE) for BEC trapped in a tight cigar-shaped potential. By means of systematic simulations, a stability region is identified in the plane of the modulation amplitude and frequency. In the low-frequency regime, solitons feature chaotic evolution, although they remain robust objects.
\end{abstract}

\section{INTRODUCTION}

Dilute atomic Bose-Einstein condensates (BECs) are accurately described by the Gross-Pitaevskii equation (GPE), alias the cubic nonlinear Schrödinger equation (NLSE) [1]. The sign of the cubic term in the GPE corresponds to the self-defocusing or focusing, if interactions between atoms in the condensate are characterized, respectively, by the positive or negative $s$-wave scattering length. The self-focusing GPE in any dimension (1D, 2D, or $3 \mathrm{D})$ gives rise to soliton solutions, which are stable in the 1D case. The creation of 1D matter-wave solitons has been reported in experimental works 2], while 2D and $3 \mathrm{D}$ solitons are unstable against the critical and supercritical collapse, respectively (these 2D states are usually called Townes solitons, TSs) [3]. It was predicted that TSs may be stabilized in the framework of the 2D GPE, without using an external potential, if the constant scattering length is replaced by a time-dependent one, that periodically changes its sign [4]. In BEC, this can be implemented by means of the Feshbach-resonance management (FRM), i.e., by applying a low-frequency ac magnetic field which acts via the Feshbach resonance 5]. This stabilization mechanism was demonstrated in optics, in terms of the transmission of a light beam through a bulk medium composed of layers with alternating signs of the Kerr nonlinearity [ 6 , and then in the framework of the 2D GPE [7, 10]. A somewhat similar technique was proposed recently, making use of a linear coupling, induced by means of a resonant electromagnetic wave, between two different hyperfine states of atoms, which feature opposite signs of the scattering length [11]. The analysis of the FRM was extended to include averaging techniques [12], generation of solitons from periodic waves [13], the stabilization of higher-order solitons [14], management of discrete arrays [15], and the case of a chirped modulation frequency [16]. However, the stabilization based on the FRM may be, strictly speaking, a transient dynamical regime, as extremely long simulations suggest that the FRM-stabilized TS may be subject to a very slow decay [8].
The stabilization of 3D solitons by means of the FRM technique alone is not possible, but stable 3D solitons were predicted in a model combining the FRM and a 1D periodic potential 9]. Similarly, the stabilization is possible when the FRM is applied in combination with a parabolic potential which strongly confines the condensate in one direction 10]. Most relevant to the experiment is the "cigar-shaped" setting, with the BEC tightly confined in two transverse directions, while the third direction remains free 2]. In the usual approximation, with the cubic nonlinearity in the corresponding 1D GPE, the analysis of the FRM in the latter setting amounts to that reported in Refs. 5]. However, if the density of the condensate is not very low, the description in terms of the cubic nonlinearity is inappropriate, the respective 1D equation taking the form of the nonpolynomial Schrödinger equation (NPSE). In particular, it admits the onset of the collapse in the self-attractive condensate in the framework of the 1D description [17]. Accordingly, a relevant problem, which is the subject of the present work, is to study the possibility of the collapse suppression by means of the FRM technique in the framework of the 1D NPSE. It is relevant to mention that the NPSE was recently used to describe Faraday waves generated in the cigar-shaped trap by a time-periodic modulation of the strength of the transverse confinement [18. We introduce the model in Section 2, and report results obtained by means of systematic numerical simulations in Section 3.

\section{THE NONPOLYNOMIAL SCHRÖDINGER EQUATION}

The normalized form of the 3D GPE with the transverse harmonic trapping potential, which acts in the $(x, y)$ plane, is

$$
\begin{aligned}
i \partial_{t} \psi= & {\left[-(1 / 2)\left(\partial_{x}^{2}+\partial_{y}^{2}+\partial_{z}^{2}\right)\right.} \\
& \left.+(1 / 2) \rho_{\perp}^{2}+V(z)+2 \pi g|\psi|^{2}\right] \psi .
\end{aligned}
$$


Here $\psi(x, y, z, t)$ is the mean-field wave function, and $\rho_{\perp}^{2} \equiv x^{2}+y^{2}$. Further, $g=2 a_{s} N / a_{\perp}$ is the nonlinearity strength, with $a_{s}$ the $s$-wave scattering length, $N$ the total number of atoms in the condensate, and $a_{\perp}=\sqrt{\hbar /\left(m \omega_{\perp}\right)}$ the confinement radius imposed by the transverse harmonic potential of frequency $\omega_{\perp}$, with $m$ the atomic mass. In Eq. (1) length and time are measured in units of $a_{\perp}$ and $\omega_{\perp}^{-1}$. As usual, $g>0$ and $g<0$ correspond to the repulsion and attraction between atoms in the BEC, respectively, and $V(z)$ is a weak axial potential, which may be present in addition to the strong transverse confinement. Being interested in the stabilization mechanism that does not require the extra potential, we set $V(z)=0$. Then, the $3 \mathrm{D}$ equation can be reduced to the NPSE by means of ansatz 17]

$$
\psi\left(\rho_{\perp}, z, t\right)=\frac{1}{\sqrt{\pi} \sigma(z, t)} \exp \left\{-\frac{r^{2}}{2[\sigma(z, t)]^{2}}\right\} f(z, t),
$$

where $1 \mathrm{D}$ wave function $f(z, t)$ is subject to the normalization condition, $\int_{-\infty}^{+\infty}|f(z, t)|^{2} d z=1$. Following Refs. [17]-[20], one can eliminate the transverse width, $\sigma^{4}=1+g|f|^{2}$, arriving at the NPSE,

$$
i \frac{\partial f}{\partial t}=\left[-\frac{1}{2} \frac{\partial^{2}}{\partial z^{2}}+V(z)+\frac{1+(3 / 2) g|f|^{2}}{\sqrt{1+g|f|^{2}}}\right] f .
$$

In the case of $g=$ const, stationary solutions are looked for as $f=\exp (-i \mu t) \Phi(z)$, where $\mu$ is the chemical potential, and real function $\Phi(z)$ obeys equation

$$
\mu \Phi=\left[-\frac{1}{2} \Phi^{\prime \prime}+V(z) \Phi+g \frac{1+(3 / 2) \Phi^{2}}{\sqrt{1+g \Phi^{2}}}\right] \Phi .
$$

Some numerical methods for simulations of the GPE and NPSE (with $g=$ const) were presented in Ref. 22].

In the case of the attractive nonlinearity, $g<0$, the form of Eq. (3) implies that the amplitude of the wave function is limited from above by a critical value,

$$
|f|^{2}<\left(|f|^{2}\right)_{\mathrm{cr}}=1 /|g|
$$

A dynamical collapse sets in, with transverse width $\sigma$ shrinking to zero and the solution developing a singularity in finite time, as $|f|^{2}$ approaches the critical value [19]. In Ref. [21], this was called two-dimensional primary collapse, as it is related to the transverse $2 \mathrm{D}$ dynamics.

In addition to the dynamical collapse, the NPSE also admits a static collapse, in the framework of stationary equation (4): for $g<0$ and $V(z)=0$, this equation admits bright-soliton solutions only below the critical value of the nonlinearity strength, $|g| \leq\left|g_{c}\right| \equiv 4 / 3$ [19]. At $|g| \leq\left|g_{c}\right|$, the axial density $|\Phi(z)|^{2}$ in the bright-soliton solution is smaller than the critical value imposed by condition (5). In Ref. [21], this kind of the collapse was called three-dimensional primary collapse, as it involves a quasi-spherical 3D soliton. With regard to the definition of $g$, this restriction determines the largest number of atoms possible in the soliton, $N<N_{\max }=\left(2 a_{\perp} / 3\left|a_{s}\right|\right)$. With $a_{\perp}$ and $\left|a_{s}\right|$ on the order of $\mu \mathrm{m}$ and $0.1 \mathrm{~nm}$, respectively, which is typical for experiments in the ${ }^{7} \mathrm{Li}$ condensate [2], one has $N_{\max } \sim 10^{4}$ atoms.

The FRM technique which makes it possible to stabilize 2D matter-wave solitons is based on the respective GPE, $i \partial_{t} \psi=\left[-(1 / 2)\left(\partial_{x}^{2}+\partial_{y}^{2}\right)+2 \pi g(t)|\psi|^{2}\right] \psi$, where constant $g<0$ is replaced by a periodic function, $g(t)=g_{\mathrm{dc}}+g_{\mathrm{ac}} \sin (\omega t)$, with $\left|g_{\mathrm{dc}}\right|<g_{\mathrm{ac}}$, so that $g(t)$ alternates between attraction and repulsion. The stabilization requires the presence of the constant ("dc") component which corresponds to the self-attraction on the average, i.e., $g_{\mathrm{dc}}<0$.

The action of the FRM within the framework of the NPSE was not considered before. To explore this situation, we take $g(t)$ as indicated above, arriving at the following modification of Eq. (3):

$$
i \frac{\partial f}{\partial t}=\left[-\frac{1}{2} \frac{\partial^{2}}{\partial z^{2}}+\frac{1+(3 / 2)\left[g_{\mathrm{dc}}+g_{\mathrm{ac}} \sin (\omega t)\right]|f|^{2}}{\sqrt{1+\left[g_{\mathrm{dc}}+g_{\mathrm{ac}} \sin (\omega t)\right]|f|^{2}}}\right] f
$$

Our objective is to identify a region in parameter space $\left\{g_{\mathrm{ac}}, g_{\mathrm{dc}}, \omega\right\}$ where the solitons subjected to the "management" represent stable solutions of Eq. (6).

\section{RESULTS}

Localized solutions to Eq. (6) were categorized as stable ones if, in direct simulations, they featured persistent pulsations, avoiding collapse or decay up to $t=5000$ (in some cases, the stability was checked up to $t=80000)$. However, the application of this criterion to the case of $\omega \lesssim 0.5$ is complicated by the fact that, under the action of the low-frequency management, the soliton tends to develop an apparently chaotic behavior, although without a trend to decay, see below. Fixing the time interval as that comprising a large number of periods, simulations become increasingly more difficult for $\omega \rightarrow 0$.

The simulations we performed by means of the CrankNicolson algorithm with open-ended boundary conditions. The initial state was $f_{0}(z)=\operatorname{sech}(z)$, that was refined by the integration of Eq. (6) with $g_{\mathrm{ac}}=0$ in imaginary time [22]. The so generated configuration was then used as the input to simulate Eq. (6) in real time.

Typical examples of stable and unstable solutions are shown in Figs. 1 and 2 (in the model with $g_{\mathrm{ac}}=0$, the respective soliton is stable). In the latter case, a gradually growing amplitude of the soliton attains critical value (5) at finite $t$, which implies the onset of the dynamical collapse. It happens when the argument of the square root in Eq. (6) becomes zero, i.e., the transverse width of the soliton shrinks to zero. Note that, with $g=g(t)$, critical density (5) of the axial wave function does not necessarily correspond to the maximum of $g(t)$. Indeed, in Fig. 2(a) the collapse happens at $g(t)$ smaller than its maximum value, $\left|g_{\mathrm{dc}}\right|+\left|g_{\mathrm{ac}}\right|$. 


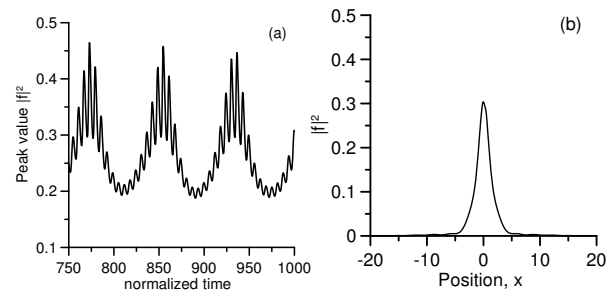

FIG. 1: A typical example of a stable soliton solution to Eq. (6). (a) The evolution of the soliton's amplitude in time. (b) A snapshot of the soliton at $t=100$. The integration step is $\Delta t=0.05$, and the size of the integration domain is $L=200$. Parameters are $g_{\mathrm{dc}}=-1, g_{\mathrm{ac}}=1.5, \omega=1$.
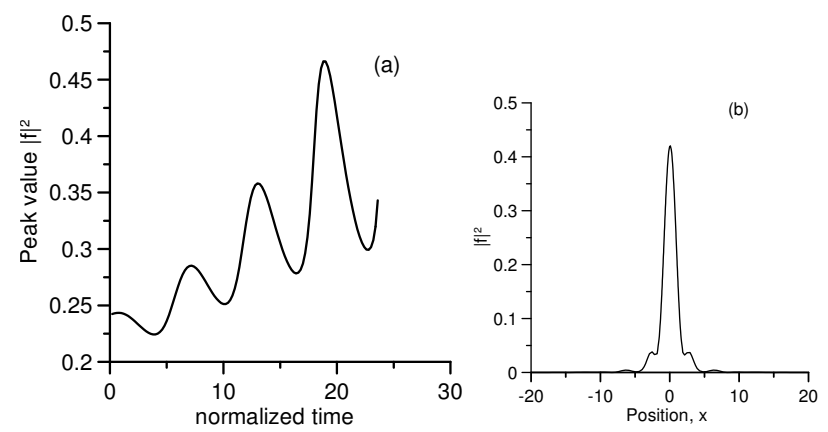

FIG. 2: A typical example of the collapsing soliton. (a) The evolution of the amplitude up to $t \approx 25$, when it reaches critical value (5). (b) A snapshot of the soliton just before the onset of the collapse. Parameters are the same as in Fig. 1 except for $g_{\mathrm{ac}}=2$.

Results of systematic simulations are summarized in the form of the stability diagram displayed in Fig. 3. The stability thresholds shown in the figure, i.e. the maximum value of $g_{\mathrm{ac}}$ admitting stable solitons, were found by slowly increasing $g_{\mathrm{ac}}$ in steps of $\Delta g_{\mathrm{ac}}=0.1$, until the instability was attained. The shape of the stability domain in the plane of the management parameters, $\left(g_{\mathrm{ac}}, \omega\right)$, is roughly similar to that which was found in management models of a different type, with the timeperiodic modulation applied not to the nonlinearity, but to the optical-lattice potential, which is necessary for the existence of stable solitons in those cases. These include the 1D model for gap solitons, with a positive scattering length [23], and the 2D GPE with a negative scattering length and $1 \mathrm{D}$ or $2 \mathrm{D}$ periodic potential, that stabilizes TSs in the respective settings [24]. As in those works, one may expect that here, at very large values of $\omega$, the stability region will start to expand in the direction of larger values of $g_{\mathrm{ac}}$, as in the limit of $\omega \rightarrow \infty$ the ac term averages to zero.

The stability borders in Fig. 3 are not extended to $\omega<0.5$, as in the region of the low-frequency modulation the solitons feature persistent but apparently chaotic evolution. In fact, the stability domain is well defined for $\omega \geq 1$, while in the intermediate region, $0.5 \leq \omega<1$, the randomness of the soliton evolution makes the stabil-

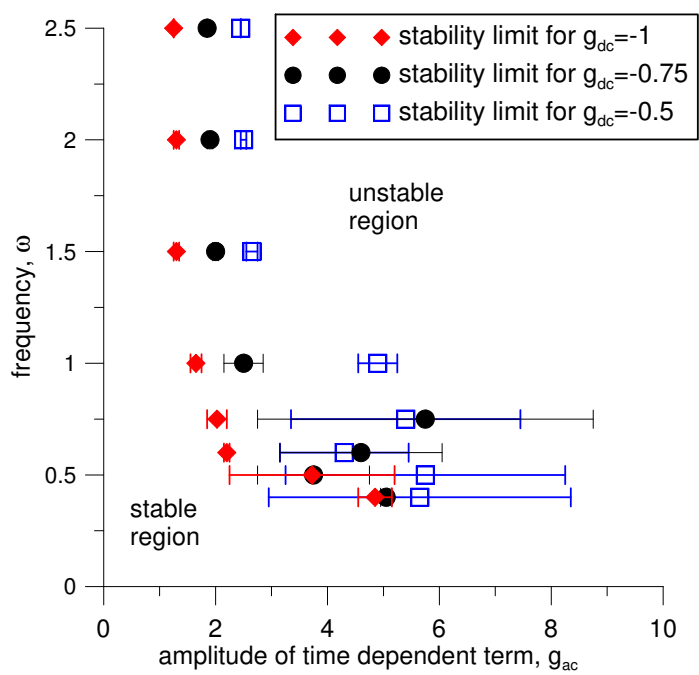

FIG. 3: (Color online). Stability borders in the plane of the time-modulation parameters, $\left(\omega, g_{\text {ac }}\right)$, as obtained from systematic simulations for different fixed values of $g_{\mathrm{dc}}$. In cases when the threshold depends upon the integration time (see Fig. (4), the respective symbol corresponds to the mean value, with the error bars given as per the respective semi-dispersion.

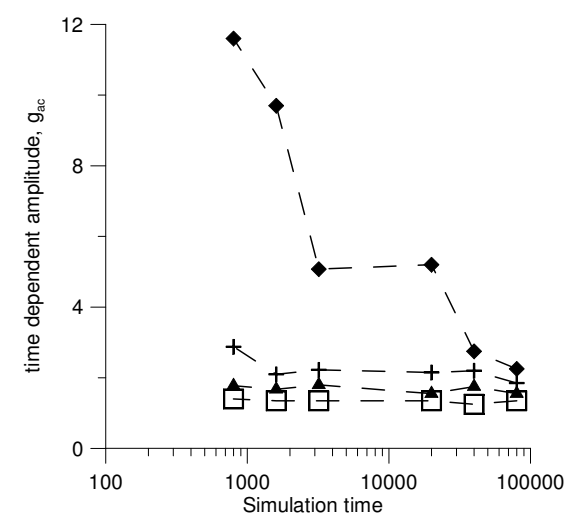

FIG. 4: Dependence of $g_{\mathrm{ac}}$ at the soliton's instability border on the integration time, for different modulation frequencies and $g_{\mathrm{dc}}=-1$. Symbols denote the stability limits at different frequencies: $\omega=0.5$ (diamonds), $=0.75$ (crosses), 1 (triangles), 1.5 (squares).

ity border dependent on the integration time - see Fig. 4. which demonstrates a natural trend to a decrease of the effective instability threshold with the increase of the evolution time, if the threshold is sensitive to it at all.

\section{CONCLUSION}

We have used the NPSE, i.e., the 1D mean-field equation for tightly trapped BEC, with the nonpolynomial nonlinearity admitting the onset of the collapse in the framework of the 1D description, for the study of the stabilization of solitons by means of the FRM (Feshbachresonant-management) technique. The results were re- 
ported in the form of stability diagrams in the plane of the management parameters, $\left(g_{\mathrm{ac}}, \omega\right)$. The stability domain is roughly similar to that reported in linearmanagement models for $1 \mathrm{D}$ gap solitons and 2D TSs (Townes solitons), supported by optical lattices subjected to the time-periodic modulation. However, stability domains of such a form have not been reported before in models of the nonlinearity management. At small values of the modulation frequency, the stability border becomes fuzzy, as solitons feature chaotic evolution in that case.
[1] A. J. Leggett, Quantum Liquids (Oxford University Press, Oxford, 2006).

[2] K. E. Strecker et al., 417, 153 (2002); L. Khaykovich et al., Science 296, 1290 (2002); S. L. Cornish, S. T. Thompson, and C. E. Wieman, Phys. Rev. Lett. 96, 170401 (2006).

[3] L. Bergé, Phys. Rep. 303, 259 (1998).

[4] B.A. Malomed, Soliton Management in Periodic Systems (Springer-Verlag, New-York, 2006).

[5] P. G. Kevrekidis, G. Theocharis, D. J. Frantzeskakis, and B. A. Malomed, Phys. Rev. Lett. 90, 230401 (2003); F. Kh. Abdullaev, R. M. Galimzyanov, M. Brtka, and R. A. Kraenkel, J. Phys. B: At. Mol. Opt. Phys. 37, 3535 (2004); G. Herring et al., Phys. Lett. A 367, 140 (2007).

[6] I. Towers and B. A. Malomed, J. Opt. Soc. Am. 19, 537 (2002).

[7] F. Kh. Abdullaev, J. G. Caputo, R. A. Kraenkel, and B. A. Malomed, Phys. Rev. A 67, 013605 (2003); H. Saito and M. Ueda, Phys. Rev. Lett. 90, 040403 (2003); G. D. Montesinos, V. M. Pérez-García, and H. Michinel, Phys. Rev. Lett. 92, 133901 (2004).

[8] A. Itin, T. Morishita, and S. Watanabe, Phys. Rev. A 74, 033613 (2006).

[9] M. Trippenbach, M. Matuszewski, and B. A. Malomed, Europhys. Lett. 70, 8 (2005); M. Matuszewski, E. Infeld, B. A. Malomed, and M. Trippenbach, Phys. Rev. Lett. 95, 050403 (2005).

[10] G. D. Montesinos, V. M. Pérez-García, and P. J. Torres, Physica D 191, 193 (2004).

[11] H. Saito, R. G. Hulet, and M. Ueda, Phys. Rev. A 76, 053619 (2007); H. Susanto, G. K. Kevrekidis, B. A. Malomed, and F. Kh. Abdullaev, Phys. Lett. A 372, 1631 (2008).

[12] D. E. Pelinovsky, P. G. Kevrekidis, and D. J. Frantzeskakis, Phys. Rev. Lett. 91, 240201 (2003); V. Zharnitsky and D. Pelinovsky, Chaos 15, 037105 (2005); M. A. Porter, M. Chugunova, and D. E. Pelinovsky, Phys. Rev. E 74, 036610 (2006)
[13] F. Kh. Abdullaev, A.M. Kamchatnov, V. V. Konotop, and V. A. Brazhnyi, Phys. Rev. Lett. 90, 230402 (2003); V. A. Brazhnyi and V. V. Konotop, Phys. Rev. A 72, 033615 (2005).

[14] A. Alexandrescu, G. D. Montesinos, and V. M. PérezGarcía, Phys. Rev. A 75, 046609 (2007).

[15] F. Kh. Abdullaev, E. N. Tsoy, B. A. Malomed, and R. A. Kraenkel, Phys. Rev. A 68, 053606 (2003); Z. Rapti, P. G. Kevrekidis, A. Smerzi, and A. R. Bishop, J. Phys. B: At. Mol. Opt. Phys. 37, S257 (2004); Y. V. Kartashov and V. A. Vysloukh, Phys. Rev. E 70, 026606 (2004); J. Cuevas, B. A. Malomed, and P. G. Kevrekidis, ibid. 71, 066614 (2005).

[16] A. I. Nicolin, M. H. Jensen, and R. Carretero-González, Phys. Rev. E 75, 036208 (2007).

[17] L. Salasnich, Laser Phys. 14, 198 (2002); L. Salasnich, A. Parola, and L. Reatto, Phys. Rev. A 65, 043614 (2002).

[18] A. I. Nicolin, M. H. Jensen, J. W. Thomsen, and R. Carretero-González, Physica D 237, 2476 (2008).

[19] L. Salasnich, A. Parola, and L. Reatto, Phys. Rev. A 66, 043603 (2002); L. Salasnich, A. Parola, and L. Reatto, Phys. Rev. A 70, 013606 (2004); L. Salasnich, Phys. Rev. A 70, 053617 (2004).

[20] L. Salasnich and B. A. Malomed, Phys. Rev. A 74, 053610 (2006); L. Salasnich, A. Cetoli, B. A. Malomed, and F. Toigo, ibid. 75, 033622 (2007); L. Salasnich et al., ibid. 76, 013623 (2007); L. Salasnich, B. A. Malomed, and F. Toigo, ibid. 76, 063614 (2007); 77, 035601 (2008).

[21] L. D. Carr and J. Brand, Phys. Rev. A 70033607 (2004).

[22] E. Cerboneschi et al., Phys. Lett. A 249, 495 (1998); L. Salasnich, A. Parola, and L. Reatto, Phys. Rev. A 64, 023601 (2001).

[23] T. Mayteevarunyoo and B. A. Malomed, Phys. Rev. A 74, 033616 (2006).

[24] T. Mayteevarunyoo, B. A. Malomed, and M. Krairiksh, Phys. Rev. A 76, 053612 (2007); G. Burlak and B. A. Malomed, ibid. 77, 053606 (2008). 